\title{
¿ES LA PÉRDIDA DE CONCIENCIA DE LA INDUCCIÓN ANESTÉSICA UN FENÓMENO PREDOMINANTEMENTE CORTICAL O TRONCAL? ¿QUÉ NOS DICE EL EXAMEN NEUROLÓGICO?
}

\author{
Pablo Sepulveda ${ }^{1}$, Ignacio Acosta ${ }^{1}$, Esperanza Carrasco ${ }^{1}$, Arnold Hoppe $^{2}$ \\ Clínica Alemana Santiago, Servicio Anestesia. \\ Clínica Alemana Santiago, Servicio Neurología.
}

Introducción: La pérdida de conciencia en Anestesia se discute si ocurre en primera instancia por pérdida de la actividad troncal o corticotalámica. Hay dos modelos explicativos: "Botom-up" priorizando actividad troncal reticular y el "Top- Down" que prioriza la pérdida de la capacidad integrativa cortical ${ }^{1}$. Si bien el fenómeno es probablemente mixto, proponemos que en anestesia la predominancia de uno u otro sería altamente dependiente de la velocidad de administración del fármaco.

Objetivo General: El objetivo de este estudio es intentar responder esta interrogante a través de una evaluación neurológica sistematizada en inducciones rápidas y lentas usando escala de coma FOUR ${ }^{2}$. (que evalúa y gradúa respuestas E oculares, $\mathrm{M}$ motoras, $\mathrm{B}$ reflejos troncales y $\mathrm{R}$ respiración).

Material y Métodos: Ensayo clínico experimental y randomizado aprobado por Comité de Ética y consentimiento informado, incluyó adultos ASA I- II, sin enfermedades neurológicas y con examen físico normal. Los pacientes fueron randomizados en dos grupos, GR (inducción rápida) con propofol en TCI efecto modelo Marsh ke0 1,21 $\mathrm{min}^{-1}$ a una diana calculada de $5.4 \mathrm{ug} /$ $\mathrm{ml}(\mathrm{EC})^{3}$ y GL (inducción lenta) $10 \mathrm{mg} / \mathrm{kg} / \mathrm{h}$ con CeCALC mismo modelo cinético. Un mismo neurólogo, ciego a la velocidad de inducción, realizó evaluaciones neurológicas cada $30 \mathrm{seg}$ con la escala de FOUR ${ }^{2}$, hasta alcanzar la pérdida de conciencia (LOC), definida por prueba de FOUR (E0 ausencia de apertura ocular y/o M0 sin respuesta motora). Al LOC se evaluó la presencia de reflejos de tronco (B ausencia pupila y corneal), patrón respiratorio ( $\mathrm{R}$ apnea), CeCALC e índice PSI SEDLINETM. Se utilizó prueba de Fisher exacta para describir, el outcome primario, y diferencias entre $\mathrm{B}$ y $\mathrm{R}$ entre grupos. $\mathrm{La}$ diferencia en los tiempos en alcanzar LOC, Ce y PSI entre grupos fue con t- Student.

Resultados: Se incluyeron 8 pacientes por grupo comparables en edad, peso y talla. La diferencia en Tiempo, Ce y PSI al LOC se ven en tabla 1. Todos los pacientes de GL tuvieron una prueba B y R de 4 puntos (reflejos de tronco indemnes), en el GR 7 pacientes tuvieron $\mathrm{B}$ y $\mathrm{R}$ de 0 puntos (reflejos de tronco abolidos) y 1 paciente tuvo $\mathrm{B}$ y R de 4 puntos (reflejos de tronco indemnes), la prueba de Fisher exacta (f 0,001 ) evidenció diferencias significativas de los reflejos de tronco al momento del LOC.

Conclusiones: Existe diferencias significativas en la presencia de reflejos troncales al momento del LOC dependiente de la forma de administración. Esto sugiere que los mecanismos fundamentales de la inconciencia anestésica se iniciarían en el córtex y no en el tronco. Esto tendría importancia en la aproximación de titulación de la inconciencia y en la definición farmacológica de lo que denominamos sitio efector.

\section{Referencias}

1. Fronters in Systems Neuroscience doi: 10.3389/fnsys.2014.0015Ann Neurol 2005;58:585-593.

2. Anesthesiology 1999; 90:92-7.

Tabla 1

\begin{tabular}{|lll|}
\hline & Grupo Lento & Grupo Rápido \\
$\begin{array}{l}\text { Tiempo al LOC } \\
\text { seg }\end{array}$ & $682(330-1.140)$ & $93(60-210)^{*}$ \\
$\begin{array}{l}\text { Ce al LOC ug/ } \\
\text { ml }\end{array}$ & $2.6(\operatorname{sd} 0,44)$ & $4,8(\operatorname{sd} 0,76) *$ \\
PSI Sedline & $67(\operatorname{sd~12)}$ & $61(\operatorname{sd} 22)$ \\
\hline
\end{tabular}

* t student significativo. 